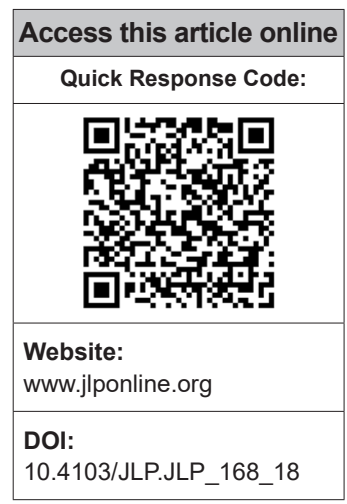

Departments of Biochemistry and ${ }^{1}$ Obstetrics and Gynaecology, Sri Ramachandra Institute of Higher Education and Research, Chennai, Tamil Nadu, ${ }^{2}$ Department of Biochemistry, JIPMER,

Puducherry, India

Address for correspondence:

Dr. Jothimalar

Ramalingam,

Department of

Biochemistry, Sri

Ramachandra Institute of Higher Education and Research, Porur,

Chennai - 600 116,

Tamil Nadu, India.

E-mail: drjothimalar@ gmail.com

Submission: 10-12-2018 Accepted: 24-08-2019

\title{
Serum soluble FMS-like tyrosine kinase-1 in ectopic pregnancy
}

\author{
Sathya Selvarajan, Jothimalar Ramalingam, Jaya Vijayaraghavan', \\ Zachariah Bobby ${ }^{2}$
}

\section{Abstract:}

CONTEXT: The diagnosis of an ectopic pregnancy (EP) requires the usage of serial beta-human chorionic gonadotropin (hCG) measurements and ultrasonography to locate the gestational sac. With the rising trends in its incidence, a rapid, noninvasive biomarker to detect this condition at the earliest can aid in decreasing the morbidity and mortality linked with EP.

AIMS: This study was performed to determine the serum level of soluble FMS-like tyrosine kinase-1 (sFLT-1) at 4-10-week gestation in EP and normal pregnancy and to identify whether it can be used as a biomarker to distinguish an EP from a normal intrauterine pregnancy.

SETTINGS AND DESIGN: This was a prospective case-control study conducted over 2 years from 2015 to 2017 in 280 women between the age groups of 19 and 38 years at a tertiary level hospital. SUBJECTS AND METHODS: Levels of SFLT-1 in sera of 140 women with EP and 140 women with normal pregnancy were assayed by a sandwich enzyme-linked immunosorbent assay at Sri Ramachandra Medical College and Research Institute, Chennai, Tamil Nadu, India.

STATISTICAL ANALYSIS USED: Statistical analyses were performed with SPSS software version 16.0, and $P \leq 0.05$ was considered statistically significant.

RESULTS: The median SFLT-1 level in EP was $419 \mathrm{pg} / \mathrm{ml}$. This was significantly lower than the value of $898 \mathrm{pg} / \mathrm{ml}$ in normal pregnancy. Receiver operating characteristic curve analysis showed that at a cutoff of $623 \mathrm{pg} / \mathrm{ml}$, sFLT-1 was able to distinguish an EP from a normal intrauterine pregnancy with a sensitivity of $98.6 \%$ and a specificity of $90.7 \%$.

CONCLUSIONS: The present study showed the significant early lowering of SFLT-1 in EP and may be considered as an effective biomarker compared to beta-hCG.

Key words:

Beta-human chorionic gonadotropin, biomarker, diagnostic test, ectopic pregnancy, gestational age, normal pregnancy, sensitivity, serum sFLT-1 concentration, specificity

\section{Introduction}

- $c$ ctopic pregnancy (EP) originated from the Greek term "ektopos," denoting out of place, and it implies the implantation of the fertilized ovum outside the intrauterine cavity. ${ }^{[1]}$ In developing nations, a greater part of hospital-related research studies on EP have stated casualty levels of about $1 \%-3 \%, 10$ times greater than those in developed nations. ${ }^{[2]}$

This is an open access journal, and articles are distributed under the terms of the Creative Commons Attribution-NonCommercial-ShareAlike 4.0 License, which allows others to remix, tweak, and build upon the work non-commercially, as long as appropriate credit is given and the new creations are licensed under the identical terms.

For reprints contact: reprints@medknow.com
The frequency of EP amid women who visit the emergency with first-trimester pain, bleeding, or both spans from $6 \%$ to $16 \%{ }^{[3]}$ The general occurrence of EP redoubled during the $20^{\text {th }}$ century, greatly linked with an augmented occurrence of pelvic inflammatory disease, artificial reproductive technologies, and increased maternal age to name a few. ${ }^{[4,5]}$

The fatality of an EP multiplies with the chance of an impending rupture. It can detonate a potentially life-threatening intra-abdominal hemorrhage and impair

How to cite this article: Selvarajan S, Ramalingam J, Vijayaraghavan J, Bobby Z. Serum soluble FMS-like tyrosine kinase-1 in ectopic pregnancy. J Lab Physicians 2019;11:335-9. 
future fertility. With the advent of high-resolution ultrasounds and highly sensitive beta-human chorionic gonadotropin (hCG) assays, there is an improvement in the diagnosis of EP, and the incidence of rupture has declined. ${ }^{[6]}$ However, the time window required for serial measurement of beta-hCG poses a delay in diagnosis. Therefore, there is a compelling demand for the advent of new, noninvasive serum tests to diagnose EP with high sensitivity and specificity to prevent not only sudden, life-threatening complications but also unnecessary medical or surgical intervention that may interrupt a potentially viable pregnancy. ${ }^{[7]}$ Numerous biomarkers of EP have been proposed, although with inadequate validation. These include markers associated with implantation and those linked to the embryo. ${ }^{[8]}$

Soluble FMS-like tyrosine kinase-1 (sFLT-1) is the soluble variant of the vascular endothelial growth factor (VEGF) receptor-1. sFLT-1 ensnares VEGF with excessive affinity, inhibiting its mitogenic activity on vascular endothelial cells, and may personate an imperative part in downregulating angiogenesis.

\section{Subjects and Methods}

This prospective case-control study was conducted from April 2015 to August 2017 in 280 women between the age group of 19 and 38 years at Sri Ramachandra Medical College and Research Institute, Tamil Nadu. One hundred and forty cases were included sequentially from patients who were willing to participate in the study, admitted with an EP between 4 and 10 weeks to the Obstetrics \& Gynaecology (OBG) inpatient department. The admitted women were followed up to confirm the diagnosis of an EP either with an ultrasound or later on when a laparotomy was performed for surgical treatment. One hundred and forty controls were sequentially included from pregnant women who agreed to participate in this study, attending their first routine hospital booking visit between 4 and 10 weeks of gestation in the OBG outpatient department.

The Institutional Ethics Committee of Sri Ramachandra Medical College and Research Institute, Chennai, Tamil Nadu, India, approved the study and was performed in accordance with its recommendations and that of the revised Helsinki Declaration. All women participating in this study gave written informed consent.

History and clinical findings of the participants were documented together with maternal age, parity, and gravidity. Gestational age was obtained from first-trimester ultrasound reports, either transvaginal or transabdominal whichever was performed for diagnosis in the women. Samples were included only if they fulfilled the following conditions: (1) female patients aged 18 years and above, (2) serum beta-hCG $\geq 5 \mathrm{IU} / \mathrm{L}$, (3) singleton gestation and (4) gestational age $\leq 10$ weeks. A venous blood sampling was carried out by a trained phlebotomist on all study participants, collected in serum separator tubes, spun at $3000 \mathrm{rpm}$ for $15 \mathrm{~min}$ and serum separated. sFLT-1 was assayed by a sandwich enzyme-linked immunosorbent assay (ELISA), Quantikine Colorimetric ELISA from R and D Systems from Minneapolis, MN, USA.

\section{Results}

The Statistical Package for the Social Sciences software version 16.0 (SPSS Inc., Chicago, IL, USA) was the statistical software used, and a two-tailed $P<0.05$ was considered statistically significant. Since the data did not follow normality, nonparametric tests were done. Median and range were calculated for the parameters using Mann-Whitney U-test. Correlation of gestational age with sFLT-1 values was performed by determining Karl Pearson's correlation coefficient. Linear regression analysis was performed to determine significant predictors of sFLT-1.

The average maternal age in normal pregnant women was $25 \pm 4$ years, and in EP, it was $26 \pm 3$ years. The average gestational age in normal pregnant women was $7 \pm 2$ weeks, and in EP, it was $6 \pm 2$ weeks. For these baseline characteristics, Student's $t$-test was used to compare between normal pregnancy and EP, and $P=0.073$ was obtained for maternal age and 0.131 for gestational age. There was no statistical difference with regard to maternal age and gestational age. Therefore, the two groups of women can be deemed to be age and gestational age matched. In this study, the median value of sFLT-1 in normal pregnancy was found to be $898 \mathrm{pg} / \mathrm{ml}$, and in EP, it was $419 \mathrm{pg} / \mathrm{ml}$; the difference was statistically significant [Table 1].

The sFLT- 1 values across various gestational ages are tabulated separately for normal and EP women in Tables 2 and 3 . There was a positive correlation between gestational age and serum sFLT-1 concentration in normal pregnancy patients [Table 2] with an $r$ value of

Table 1: Comparison of soluble FMS-like tyrosine kinase-1 and beta-human chorionic gonadotropin between normal pregnancy and ectopic pregnancy

\begin{tabular}{lcc}
\hline \multirow{2}{*}{ sFLT-1 $(\mathrm{pg} / \mathrm{ml})$} \\
\cline { 2 - 3 } & Normal pregnancy & EP \\
\hline Median (IQR) & $898(745-1371)$ & $419(315-471)$ \\
Minimum & 163 & 63 \\
Maximum & 15,837 & 835 \\
$P$ & \multicolumn{2}{c}{0.000} \\
\hline
\end{tabular}

IQR=Interquartile range, sFLT-1=Soluble FMS-like tyrosine kinase-1, $\mathrm{EP}=$ Ectopic pregnancy 
Table 2: Comparison of soluble FMS-like tyrosine kinase-1 values across gestational age for normal pregnancy and the calculated values from the regression equation for the same

\begin{tabular}{lccccc}
\hline Gestational age (weeks) & Number of cases & sFLT-1 $(\mathbf{p g} / \mathbf{m l})$, mean \pm SD & Minimum & Maximum & Calculated sFLT-1 \\
\hline 4 & 15 & $873 \pm 508$ & 496 & 2572 & 660 \\
5 & 34 & $839 \pm 350$ & 332 & 1817 & 754 \\
6 & 38 & $967 \pm 336$ & 526 & 2012 & 848 \\
7 & 25 & $1007 \pm 390$ & 163 & 2127 & 942 \\
8 & 13 & $1408 \pm 182$ & 963 & 1783 & 1036 \\
9 & 7 & $3532 \pm 5426$ & 1372 & 15,837 & 1131 \\
10 & 8 & $1777 \pm 375$ & 1288 & 2585 & 1225 \\
\hline
\end{tabular}

sFLT-1=Soluble FMS-like tyrosine kinase-1, SD=Standard deviation

Table 3: Comparison of soluble FMS-like tyrosine kinase-1 values across gestational age for ectopic pregnancy women

\begin{tabular}{l|c|c|c|c}
\hline $\begin{array}{l}\text { Gestational } \\
\text { age (weeks) }\end{array}$ & $\begin{array}{c}\text { Number } \\
\text { of cases }\end{array}$ & $\begin{array}{c}\text { sFLT-1 }(\mathbf{p g} / \mathrm{ml}), \\
\text { mean } \pm \text { SD }\end{array}$ & Minimum & Maximum \\
\hline 4 & 3 & $402 \pm 122$ & 342 & 543 \\
5 & 32 & $403 \pm 85$ & 168 & 631 \\
6 & 50 & $422 \pm 118$ & 63 & 585 \\
7 & 19 & $396 \pm 112$ & 123 & 563 \\
8 & 17 & $487 \pm 169$ & 176 & 835 \\
9 & 12 & $274 \pm 44$ & 192 & 350 \\
10 & 7 & $180 \pm 56$ & 63 & 243 \\
\hline
\end{tabular}

0.316 statistically significant at $P=0.000$. On comparing the sFLT-1 values between controls and EP across gestational age, there was an obvious difference present as indicated in Table 4 . The sFLT- 1 concentration in EP is always $1 / 2-1 / 3^{\text {rd }}$ of the corresponding week of gestation in normal pregnancy [Figure 1], but the number of samples was insufficient for statistical calculation and hence was not performed. Receiver operating characteristic (ROC) curve analysis [Figure 2] revealed that at a cutoff of $623 \mathrm{pg} / \mathrm{ml}$, sFLT-1 was able to distinguish an EP from a normal pregnancy with a sensitivity of $98.6 \%$ and a specificity of $90.7 \%$.

A multiple linear regression was calculated to predict sFLT-1 values based on gestational age and the nature of the pregnancy whether normal or ectopic. The regression equation for predicting the sFLT-1 level based on the gestational age is equal to $282.57+94.226$ (gestational age in weeks). sFLT-1 increased $94.226 \mathrm{pg} / \mathrm{ml}$ for each gestational week from 4 to 10 weeks in normal pregnancy. Hence, the values obtained can be used for predicting EP.

\section{Discussion}

In normal pregnancy, enormous quantities of VEGF are secreted by the macrophages at the Nitabuch's stria of decidua during the first trimester of pregnancy. ${ }^{[9]}$ This is where the process of vascular transformation is necessitated instead of angiogenesis. A balance between angiogenesis and vascular transformation is

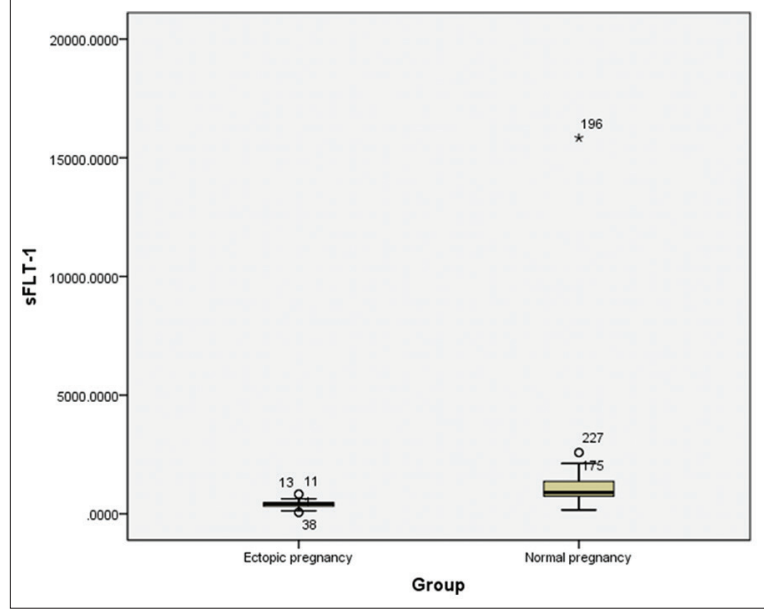

Figure 1: Box plot showing comparison of sFLT-1 values in ectopic pregnancy and normal pregnancy

therefore necessary. Therefore, sFLT-1 may be involved in neutralizing the influence of VEGF on maternal endothelial cells in the decidua. sFLT-1 mRNA is produced by the villous trophoblast in substantial amounts all through pregnancy. ${ }^{[10]}$ Since there is a dramatic increase in the size of the placenta and in consequence that of the villous trophoblast in the course of pregnancy, it is to be expected that the overall sFLT-1 production will increase.

sFLT-1 is produced by the trophoblastic cells of the placenta which are positioned between the mother's blood vessels on one side and the umbilical vessels on the side of the fetus, ${ }^{[11]}$ thereby advocating that sFLT-1 traps and binds VEGF and placental growth factor forming a barricade in opposition to atypical vascular penetrability and aberrant angiogenesis, for instance, the merging of fetal blood vessels to maternal capillaries. The trophoblastic villi have an uninterrupted communication with maternal circulation within the placenta, and therefore, the proteins produced there can be identified in maternal blood.

Since the implantation of EP at the tubal site is unfavorable, it provides an abnormal environment with insufficient nutrition and oxygen to the developing embryo. This hypoxic environment increases the expression of VEGF 
Table 4: Comparison of soluble FMS-like tyrosine kinase-1 values between controls and ectopic pregnancy across gestational age

\begin{tabular}{|c|c|c|c|c|}
\hline \multirow{2}{*}{$\begin{array}{l}\text { Gestational age } \\
\text { (weeks) }\end{array}$} & \multicolumn{2}{|c|}{ Normal pregnancy } & \multicolumn{2}{|r|}{ EP } \\
\hline & Number of cases & sFLT-1 (pg/ml), mean $\pm S D$ & Number of cases & sFLT-1 (pg/ml), mean \pm SD \\
\hline 4 & 15 & $873 \pm 508$ & 3 & $402 \pm 122$ \\
\hline 5 & 34 & $839 \pm 350$ & 32 & $403 \pm 85$ \\
\hline 6 & 38 & $967 \pm 336$ & 50 & $422 \pm 118$ \\
\hline 7 & 25 & $1007 \pm 390$ & 19 & $396 \pm 112$ \\
\hline 8 & 13 & $1408 \pm 182$ & 17 & $487 \pm 169$ \\
\hline 9 & 7 & $3532 \pm 5426$ & 12 & $274 \pm 44$ \\
\hline 10 & 8 & $1777 \pm 375$ & 7 & $180 \pm 56$ \\
\hline
\end{tabular}

sFLT-1=Soluble FMS-like tyrosine kinase-1, EP=Ectopic pregnancy, SD=Standard deviation

Table 5: Sensitivity and cutoff values of soluble FMS-like tyrosine kinase-1 at various specificities

\begin{tabular}{lcc}
\hline Specificity, \% & Sensitivity, \% & Cutoff value of sFLT-1 $(\mathrm{pg} / \mathrm{ml})$ \\
\hline 90.0 & 98.6 & 634 \\
92.1 & 95.0 & 582 \\
93.6 & 90.0 & 542 \\
95 & 87.9 & 527 \\
\hline
\end{tabular}

sFLT-1=Soluble FMS-like tyrosine kinase-1

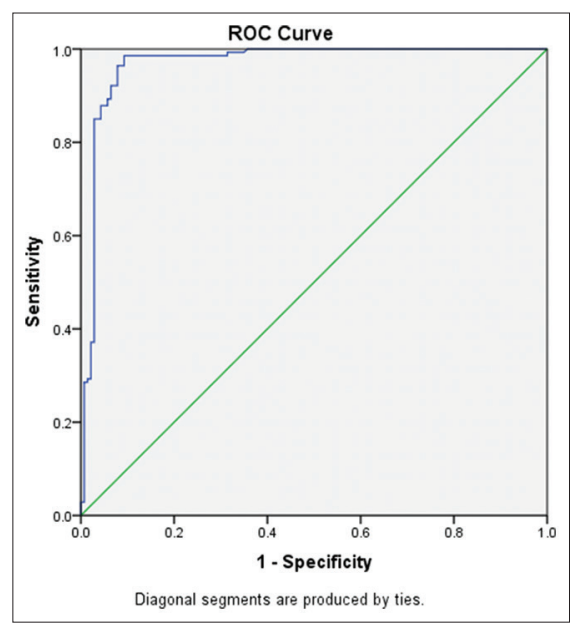

Figure 2: Receiver operating characteristic curve analysis of sFLT-1

in the ectopic site. This pro-angiogenic growth factor and its receptors were increased in production in EP. ${ }^{[12]}$ It has been identified that VEGF in serum of women with EP is elevated in comparison with intrauterine pregnancy. ${ }^{[13]}$ There is also a reduction in the levels of sFLT-1 in EP women, but the exact mechanism behind the decline in sFLT-1 is unclear. It may be either due to the subsequent binding of sFLT-1 receptor to the excessively expressed VEGF or a decrease in sFLT-1 production or both. Measurement of sFLT-1, therefore, aids in identifying implantation of the embryo at an ectopic site and can, therefore, be used as a biomarker for EP.

In this study, the median value of sFLT-1 in normal pregnancy was found to be $898 \mathrm{pg} / \mathrm{ml}$. Daponte et al. in their study showcased the median sFLT-1 in normal pregnant women as $1390.32 \pm 655.37 \mathrm{pg} / \mathrm{ml} .{ }^{[14]}$ However, the sFLT-1 values reported by Martínez-Ruiz et al. were lower at $505(121-945) \mathrm{pg} / \mathrm{ml}^{\left[{ }^{[15]}\right.}$ This could be due to the fact that the population studied and the assay kit used are different in these studies.

In the EP group, the median sFLT-1 concentration was $419 \mathrm{pg} / \mathrm{ml}$, which was nearly half of that in normal population. In the Martínez-Ruiz et al. study, the median sFLT-1 value in EP was 84 (65-96) $\mathrm{pg} / \mathrm{ml}$. In the Daponte et al. study, the mean value for failed pregnancies (including EP and missed abortions) was $288.79 \pm 375.76 \mathrm{pg} / \mathrm{ml}$. Irrespective of the difference in mean sFLT-1 in normal pregnancy quoted in various studies, there is a decrease in the EP group by $75 \%-80 \%$ and was statistically significant.

The relationship between gestational age and sFLT-1 values was determined using Karl Pearson's correlation coefficient. The $r$ value for week wise correlation in normal pregnancy was 0.316 . On comparing the sFLT-1 values between controls and EP across gestational age, there was an obvious difference present [Figure 1], but the number of samples was not sufficient for statistical calculation. Gestational age is a significant predictor of sFLT-1. ROC curve analysis [Figure 2] revealed that at a cutoff of $623 \mathrm{pg} / \mathrm{ml}$, sFLT-1 was able to distinguish an EP from a normal pregnancy with a sensitivity of $98.6 \%$ and a specificity of $90.7 \%$. On fixing the specificity to higher levels of up to $95 \%$, the various sensitivities and cutoff values for sFLT- 1 are tabulated in Table 5.

An increased sample size in each gestational age is required to establish a reference interval according to the gestational age. The sFLT- 1 values of ectopic patients can be compared with gestational age-specific normal values, and the statistical significance of the difference can be established.

Since sFLT-1 is a marker of angiogenesis, it is expected to be altered in nonviable intrauterine pregnancy (IUP) also. Apart from differentiating a normal IUP from an EP, cutoff levels for sFLT-1 need to be established for differentiating EP from nonviable IUPs as well. ${ }^{[16]}$ This objective has been taken up in the past in studies by Martínez-Ruiz et al. ${ }^{[15]}$ and Daponte et al. ${ }^{[14]}$ in a limited 
sample size. A study with adequate samples in each gestational age can throw further light on this subject.

The time required for manually assaying sFLT-1 by ELISA method is more than $4 \mathrm{~h}$. Since EP is an emergency condition, a biomarker should have a shorter turnaround time and testing in an automated system. If these two criteria are met, this promising biomarker can be clinically evaluated and used as a diagnostic test for EP.

\section{Conclusion}

In conclusion, sFLT-1 was able to distinguish an EP from a normal pregnancy at a cut-off of $623 \mathrm{pg} / \mathrm{ml}$ with a good sensitivity of $98.6 \%$ and specificity of $90.7 \%$. Further study is required to determine gestational age specific reference intervals so that its clinical utility in diagnosing EP can be established.

\section{Acknowledgment}

The authors thank the Indian Council of Medical Research, New Delhi, for providing financial assistance in the form of the TSS fellowship number PhD (Integrated)-30-F.T./I/2014.

\section{Financial support and sponsorship Nil.}

\section{Conflicts of interest}

There are no conflicts of interest.

\section{References}

1. Walker JJ. Ectopic pregnancy. Clin Obstet Gynecol 2007;50:89-99.

2. Berg CJ, Callaghan WM, Syverson C, Henderson Z. Pregnancy-related mortality in the United States, 1998 to 2005. Obstet Gynecol 2010;116:1302-9.

3. Alkatout I, Honemeyer U, Strauss A, Tinelli A, Malvasi A, Jonat W, et al. Clinical diagnosis and treatment of ectopic pregnancy. Obstet Gynecol Surv 2013;68:571-81.

4. Li C, Zhao WH, Zhu Q, Cao SJ, Ping H, Xi X, et al. Risk factors for ectopic pregnancy: A multi-center case-control study. BMC Pregnancy Childbirth 2015;15:187.

5. Zhang D, Shi W, Li C, Yuan JJ, Xia W, Xue RH, et al. Risk factors for recurrent ectopic pregnancy: A case-control study. BJOG 2016;123 Suppl 3:82-9.

6. Condous G, Van Calster B, Kirk E, Haider Z, Timmerman D Van Huffel S, et al. Prediction of ectopic pregnancy in women with a pregnancy of unknown location. Ultrasound Obstet Gynecol 2007;29:680-7.

7. Barnhart KT, Katz I, Hummel A, Gracia CR. Presumed diagnosis of ectopic pregnancy. Obstet Gynecol 2002;100:505-10.

8. Rajendiren S, Dhiman P. Biomarkers of ectopic pregnancy-present and future. In: Darwish A, editor. Contemporary Gynecologic Practice. India: In Tech; 2015. p. 2.

9. Zarezade N, Saboori Darabi S, Ramezanali F, Amirchaghmaghi E, Khalili G, Moini A, et al. mRNA expression of VEGF and its receptors in fallopian tubes of women with ectopic pregnancies. Int J Fertil Steril 2015;9:55-64.

10. Clark DE, Smith SK, He Y, Day KA, Licence DR, Corps AN, et al. A vascular endothelial growth factor antagonist is produced by the human placenta and released into the maternal circulation. Biol Reprod 1998;59:1540-8.

11. Shibuya M. Vascular endothelial growth factor and its receptor system: Physiological functions in angiogenesis and pathological roles in various diseases. J Biochem 2013;153:13-9.

12. Lam PM, Briton-Jones C, Cheung CK, Leung SW, Cheung LP, Haines C. Increased messenger RNA expression of vascular endothelial growth factor and its receptors in the implantation site of the human oviduct with ectopic gestation. Fertil Steril 2004;82:686-90.

13. Felemban A, Sammour A, Tulandi T. Serum vascular endothelial growth factor as a possible marker for early ectopic pregnancy. Hum Reprod 2002;17:490-2.

14. Daponte A, Pournaras S, Polyzos NP, Tsezou A, Skentou H, Anastasiadou F, et al. Soluble FMS-like tyrosine kinase-1 (sFlt-1) and serum placental growth factor (PIGF) as biomarkers for ectopic pregnancy and missed abortion. J Clin Endocrinol Metab 2011;96:E1444-51

15. Martínez-Ruiz A, Sarabia-Meseguer MD, Pérez-Fornieles J, Vílchez JA, Tovar-Zapata I, Noguera-Velasco JA. Placental growth factor, soluble fms-like tyrosine kinase 1 and progesterone as diagnostic biomarkers for ectopic pregnancy and missed abortion. Clin Biochem 2014;47:844-7.

16. Richardson A, Deb S, Campbell B, Raine-Fenning N. Serum concentrations of Ang-2 and Flt-1 may be predictive of pregnancy outcome in women with pregnancies of uncertain viability: A phase I exploratory prognostic factor study. J Obstet Gynaecol 2018:38:321-6 